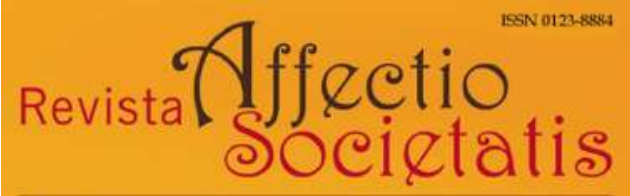

Departamento de Psicoanálisis | Universidad de Antioquia

Revista Affectio Societatis

Departamento de Psicoanálisis

Universidad de Antioquia

revistaaffectiosocietatis@udea.edu.co

ISSN (versión electrónica): 0123-8884

Colombia 


\title{
UNA TERAPÉUTICA PSICOANALITICA AL TRINOMIO Y CASUÍSTICA DE PARENTALIDADES ${ }^{1}$
}

\author{
Omar Domínguez Díaz² \\ Universidad Autónoma de San Luis Potosí, México \\ odominguezdiaz@gmail.com \\ https://orcid.org/0000-0003-2034-4524 \\ Ma. Antonia Reyes Arellano 3 \\ Universidad Autónoma de San Luis Potosí, México \\ ma.reyes.are@gmail.com \\ https://orcid.org/0000-0002-2557-097X
}

DOI: 10.17533/udea.affs.v17n33a02

\section{Resumen}

A partir de la puesta en marcha de un dispositivo psicoanalítico aplicado a la terapéutica en un hospital y mediante la investigación en psicoanálisis, se examinan los lazos psíquicos parentales y los enigmas que se esta- blecen entre los involucrados alrededor del nacimiento de un niño o durante el embarazo. Al mismo tiempo se describe el dispositivo clínico de acompañamiento durante situaciones conflictivas en dicho pasaje hos-

1 Este artículo se deriva de una investigación denominada "Acompañamiento del trinomio en el pasaje hospitalario: Una escucha psicoanalítica" finalizada en el 2018 en un posgrado de Maestría en Psicología con Área de formación profesional (AFP) en psicoanálisis: Teoría y clínica en la Universidad Autónoma de San Luis Potosí (UASLP) bajo los auspicios del Consejo Nacional de Ciencia y Tecnología (CONACYT) en México. La casuística y documentación fue realizada por Omar Domínguez, las reflexiones y elaboración de este articulo fueron compartidas con Ma. Antonia Reyes Arellano.

2 Maestrante de la Universidad Autónoma de San Luis Potosí (UASLP). Licenciado en Psicología, formado en clínica psicoanalítica.

3 Profesora investigadora de la Universidad Autónoma de San Luis Potosí. Doctora en Ciencias Humanas Clínicas por la Universidad Paris 7, Denis Diderot. Psicoanalista. 
pitalario, contribuyendo a la elaboración de las manifestaciones psíquicas asociadas a procesos orgánicos dolorosos. La casuística documentada psicoanalíticamente sobre el trinomio, forma de denominar al conjunto formado por el hijo recién nacido, su cuidador primario y el secundario, en su travesía con los agentes hospitalarios muestra que la parentalidad puede leerse desde las marcas psíquicas que se generan desde el registro de lo real.

Palabras clave: dispositivo psicoanalítico; hospital; parentalidad, embarazo; trinomio.

\section{A PSYCHOANALYTICAL THERAPY TO THE TRINOMIAL AND CASUISTRY OF PARENTALITIES}

\section{Abstract}

From the implementation of a psychoanalytic device applied to the therapy in a hospital and through research in psychoanalysis, this paper examines the parental psychic ties and the enigmas that are established within those involved around the birth of a child or during pregnancy. At the same time, it describes the clinical device of accompaniment during conflictive situations in such hospital passage, contributing to the elaboration of the psychic manifestations associ- ated with painful organic processes. The psychoanalytically documented casuistry on the trinomial -a form of denomination to the group formed by the newborn and their primary and secondary caretakers- in its journey with the hospital agents shows that parentality can be read from the psychic marks generated from the real order.

Keywords: psychoanalytic device, hospital, parentality, pregnancy, trinomial.

\section{UNE THÉRAPEUTIQUE PSYCHANALYTIQUE POUR LA TRIADE ET LA CASUISTIQUE DE PARENTALITÉS}

\section{Résumé}

À partir de la mise en place dıun dispositif psychanalytique appliqué à la thérapeutique en milieu hospitalier et à travers la recherche en psychanalyse, cet article examine les liens psychiques parentaux et les énigmes qui srétablissent entre les acteurs autour de la naissance d >un 
enfant ou pendant la grossesse. En même temps, le dispositif clinique draccompagnement lors de situations conflictuelles dans ce passage hospitalier est décrit, contribuant à leélaboration des manifestations psychiques associées aux processus organiques douloureux. La casuistique psychanalytiquement documentée sur la triade, manière dont on nomme l`ensemble formé par le nouveau-né, son soigneur primaire et le secondaire, dans son parcours avec les agents hospitaliers montre que la parentalité peut être lue à partir des marques psychiques qui sont générées par le registre du réel.

Mots-clés : dispositif psychanalytique ; hôpital ; parentalité ; grossesse ; triade.

\section{UMA TERAPÊUTICA PSICANALÍTICA AO TRINÔMIO E À CASUÍSTICA DE PARENTALIDADES}

\section{Resumo}

A partir da implementação dum dispositivo psicanalítico aplicado à terapêutica num hospital e por meio de pesquisas em psicanálise, examinam-se os laços psíquicos parentais e os enigmas que se estabelecem entre os envolvidos em torno do nascimento dum filho ou durante a gravidez. Ao mesmo tempo, é descrito o dispositivo clínico de acompanhamento durante situações de conflito naquela passagem hospitalar, contribuindo para a elaboração das manifestações psíquicas associadas a processos or- gânicos dolorosos. A casuística psicanaliticamente documentada sobre o trinômio, uma forma de nomear o grupo formado pelo recém-nascido, seu cuidador principal e o cuidador secundário, em sua jornada com os agentes hospitalares mostra que a parentalidade pode ser lida a partir das marcas psíquicas que são geradas a partir do registro do real.

Palavras-chave: dispositivo psicanalítico; hospital; parentalidade, gravidez; trinômio. 


\section{Desarrollo}

\section{Psicoanálisis y medicina}

Cada disciplina presenta y argumenta dispositivos para entender ciertos fenómenos. Quienes operan desde la medicina tienen dispositivos para observar y auscultar lo que sucede en el organismo, es lo que denominan "signos" o manifestaciones objetivas observadas en la exploración médica, clínicamente fiables. El psicoanálisis, por su parte, implementa el dispositivo que propone escuchar las referencias subjetivas, sus síntomas, las percepciones anómalas, siniestras u ominosas, así como las manifestaciones de sufrimiento o dolor en la dimensión orgánica, pero sobre todo en la esfera psíquica. La estrecha vinculación entre lo orgánico y lo psíquico da paso a que gran parte de los pensamientos inconscientes o reprimidos mantengan su correlato en la parte orgánica. Lo anterior sostiene la creación de un dispositivo de escucha psicoanalítica que atiende y contribuye a la cura durante el pasaje hospitalario de aquello de lo que los médicos no se alcanzan a ocupar.

En efecto, el psicoanálisis, invento de Freud, genera una curación distinta; tal tránsito propuso el pasaje de la auscultación y mirada del médico neurólogo a la auscultare atenta del discurso de sus pacientes. Auscultare en latín significa "escuchar con atención" (Anders, et al., s.f.), esta vez enigmas "otros" en su valor sintomático, elementos subjetivos en el decir del paciente.

A entender que lo que aqueja estrictamente al cuerpo orgánico de los pacientes, es atendido por la medicina, pero también en ocasiones requiere al psicoanálisis. Cordier (citado en Miller, 2012), una médico nutrióloga y analizante, sintetiza lo que conformaría el trabajo de un médico pasador-de-síntomas, -pasador en dos sentidos, el primero, que cristaliza un síntoma médico atribuible a la enfermedad y por lo tanto exterior al consultante en algo propio, y el segundo, que pasa los síntomas de un médico a un analista-. Para ella "hay pacientes que no están preparados para plantearse unas preguntas. Las podemos plantear a algunos, y constatar que no tienen ganas de responder. En 
tal caso, la nutrición, efectivamente, pasa a un primer plano" (p. 104). Dicho de otra forma, las preguntas analíticas, o la división subjetiva, solo convoca a ciertas personas, punto de división entre el paciente de la medicina y el analizante. Lo anterior bajo la propuesta de que el médico, como afirma Cordier, "tiene la posibilidad de restituir al síntoma su dignidad", es decir, hacerlo valer en tanto a su sentido de propio, en tanto su condición de real y emanado de lo humano, dejando de adjudicarlo como algo ajeno o susceptible de desaparecer y así volverse un "médico-pasador, pasador de síntoma, para permitir al sujeto plantearse su pregunta" (p. 48). Paradigma que conmovería a los pacientes de la medicina hacia el deseo de saber, pasaje ineludible en la división subjetiva, para el caso, que le permite a esta nutrióloga francesa identificar su hacer; mi trabajo, dice, "es médico, pero también es un trabajo preliminar al análisis" (p. 104). Por tanto, el médico-pasador de síntomas debe ocupar el lugar de la persona que cuestione sobre la situación, así el paciente debería salir del consultorio médico con dudas y entrar al consultorio analítico para intentar resolverlas. Dicho diagnóstico diferencial posiciona a cada paciente con una demanda determinada: de curación médica o de análisis.

Esta es una nueva vertiente que está tomando la medicina antes descrita y que se configura en contra partida a la medicina de acción medicalizante; Kishore (citado en Orueta et al., 2002) la piensa como "la conversión en procesos patológicos de situaciones que son y han sido siempre completamente normales y el pretender resolver mediante la medicina situaciones que no son médicas sino sociales, profesionales o de las relaciones interpersonales" (p. 151), se enquista en una sola y única ideología de cura, evitando pensar los procesos afectivos en los neonatos, es decir, casos "para los que la medicina no dispone de soluciones adecuadas ni eficaces" (p. 151).

El médico es causa de la medicalización, cómo lo comprende la pediatra Giangaspro (citado en Udenio, 2016). Por citar algunas formas en las que se lleva a cabo la medicalización, esta autora enumera las siguientes prácticas tal como comprende la cuestión:

- en razón de su formación, centra el origen de todo enfermar en su vertiente biológica, despreciando la subjetividad; 
- mantiene una actitud paternalista;

- argumenta que la razón de su modo de entender la demanda de los pacientes es la sobrecarga de trabajo; acude a la utilización de pruebas y fármacos por su dificultad para sostener las incertidumbres del diagnóstico;

- sobreutiliza nuevas tecnologías que aumentan la sensibilidad de la identificación de alteraciones de la normalidad y reducen el umbral de la intervención;

- tiende a la participación en la innovación terapéutica, empleando nuevos fármacos sin pruebas que aporten avances destacables respecto de los ya existentes;

- realiza una medicación defensiva, más pruebas y más tratamientos "por si acaso", por temor a posibles denuncias;

- interviene, según el inmediatismo vigente, sobre cualquier consulta, cualquiera sea el motivo de la misma;

- condiciona algunas decisiones a las relaciones establecidas con la industria farmacéutica, cuyo conocido objetivo son los máximos beneficios, no importa cuáles sean las estrategias. (pp. 23-24).

Una adolescente de 17 años de edad con embarazo de alto riesgo, y diabetes juvenil, que había perdido a su hijo en el $5^{\circ}$ mes de embarazo y que amenaza a las enfermeras con suicidarse, dice estar desecha por la pérdida de lo único que le daba esperanza en su vida. La cantidad de consumo del medicamento que se le administró para provocar la expulsión del feto preocupa al equipo de cuidado, de manera tal que se encuentra al límite de lo permitido, situación médica inexplicable. Si se le proporcionara una dosis más alta del medicamento pondría en riesgo su salud. La consigna médica estima 48 horas para expulsar al producto y si no ocurre se le tendrá que intervenir mediante un legrado. La solicitud de interconsulta para el psicoanalista es dar atención frente un riesgo de suicidio.

Lo anterior nota la primacía de la vertiente biológica en la atención y el desprecio a la subjetividad y sus efectos sobre el cuerpo, al mismo tiempo es la muestra de actitudes paternalistas y de la incer- 
tidumbre del diagnóstico, ante lo cual se resalta el recurso de pruebas, fármacos y cirugías, es decir, la intensificación de la intervención médica vigoriza la intromisión a ultranza en el cuerpo del otro, como actos defensivos sostenidos por el temor de posibles denuncias.

Le conmueve a la paciente haber sentido a su hijo moverse en su vientre, situación de gran ilusión y prueba de que alguien estaba ahí para ella. Era un orgullo formar una familia. Y repetía constantemente "...y ahora no lo tengo", encajándose las uñas en las manos, lastimándose. Haciendo referencia a las pastillas abortivas o al aborto mismo declara: "ellos me lo quieren quitar". La intervención fue tomando el camino del reconocimiento de su deseo de ser madre, ahora interrumpido, pero donde las huellas de ese deseo se apalabraban, acompañándola.

Contrario a las formas que se llevan a cabo en la medicalización, aquí se trata del valor de la escucha subjetiva. Así, fue importante hablar de un hijo y no únicamente de un producto o un embarazo de riesgo que se había interrumpido, sino que ahí había una madre que necesitaba ser nombrada como tal. Fue esencial nombrarla como pareja de su novio, mujer que amaba, que no podía dar la cara por sentir que había fallado pero que su pareja no culpabilizaba. Madre que cargaría junto a ese padre a un hijo, fenecido y ya nombrado. Después de otra consulta había podido desprenderse de ese cuerpo muerto, ahora lo llevaba simbólicamente, ya no era necesario resguardarlo físicamente. Lo sucedido en esta mujer puede ser comprendido desde el movimiento de su hijo como objeto, pasado de un plano imaginario a un plano simbólico.

Lacan (1966) había denunciado con el nombre de falla epistemosomática, en su conferencia "Psicoanálisis y medicina", que esta última pretende tomar al cuerpo como un registro purificado que puede ser enteramente "fotografiado, radiografiado, calibrado, diagramado y posible de condicionar" (p. 14), dejando de lado la dimensión del goce. En ese orden de ideas es que Giangaspro propone:

intentar un abordaje de la medicalización de la vida cotidiana desde otro modo de ser médico, desde otra forma de relación médico 
paciente, en la que la verdadera función del médico sitúe en lo que le falta a su saber para dar cuenta de los acontecimientos en el cuerpo hablante $(2016$, p. 26).

Es decir, para no quedar atrapado del lado del registro purificado, contemplando así la noción de síntoma y goce.-En cuanto al síntoma, para Bassols (citado en Udenio et al., 2016, p. 15):

no sigue una evolución lineal, sino que es siempre producto de una retroacción sobre la neurosis infantil, de modo que con el sínthoma -ya entonces con th- lo que hacemos es reescribir de alguna manera aquello que fuimos como niños, no como un ideal de los otros, no como el ideal esperado por los padres, sino como una forma de goce marcada desde el inicio en el cuerpo hablante del sujeto.

Ante la especificidad de estos síntomas es importante argumentar y actuar desde la relación epistemosomática propuesta también en "Psicoanálisis y medicina" (Lacan, 1966), donde aparece la posibilidad de acompañar la medicina junto al psicoanálisis, diagnosticando de ambos lados y proponiendo tratamientos conjuntos o continuos que terminen siempre en el posicionamiento de un sujeto frente a un sinthome.

\section{De lazo binomial al lazo trinomial parental en el hospital:}

\section{enigmas, lazos, funciones y el complejo familiar}

De los lazos psíquicos parentales como de los enigmas que se establecen entre los involucrados alrededor del nacimiento de un niño o durante el embarazo, trata el siguiente apartado. El trinomio, forma de denominar al conjunto formado por el hijo recién nacido, su cuidador primario y el secundario, en su travesía con los agentes hospitalarios, intenta mostrar que la parentalidad puede leerse desde las marcas psíquicas que se generan desde el registro de lo real.

Lo anterior ha llevado a conceptualizar algunas relaciones de conformación al trinomio y sirvió para nombrar lo que el hospital crea: el proceso embarazo-nacimiento (binomio por la unión de una mujer embarazada y su hijo o hija) solicita en un primer momento la 
presencia de la madre con su hijo y una persona que se haga cargo del papeleo y los trámites, personaje que completa el trinomio. Saber que desde lo administrativo no puede darse de alta a una madre si no cuenta con una tercera persona que firme responsivamente por ella. Si el niño tiene situaciones que hagan peligrar su vida antes o después de la salida del hospital, la madre y/o el padre tienen que asistir a visitas en las unidades de cuidado infantil y es muy común ver a otra persona hacerse cargo de los trámites, completando igualmente el trinomio. En los contados casos donde la madre se presentaba a ver a su hijo y no asistía acompañada, era muy común escucharlos referenciar al bebé con una tercera persona: "mi papá tenía muchas ganas de conocerte". En otros, donde imposibilitada por el seguimiento médico o psicoanalítico no podía ver a su hijo, el padre se ocupaba de ello: "tu mamá ya te quiere conocer". En los casos anteriores termina por formarse un trinomio, pero un miembro de dicho trinomio queda invisibilizado para recibir atención o cuidados.

Visibilizar al tercero (tercera persona) aportó elementos importantes para reconocer los inter-juegos en el trinomio y los cuidados, su calidad, interés o desapego en el lazo con el neonato hospitalizado en la UCIN. Los casos donde las madres se mantenían alejadas de sus hijos por situaciones que las incapacitaban física o psíquicamente para asistirlos, se tornaban en cuidadores secundarios, permitiendo al padre sostener una función materna. En tales casos se descuidaba la atención psíquica que requerían las madres por quedar fuera del binomio que el hospital reconocía. También fue claro que cuando el padre no se acercaba a su hijo, en un registro imaginario, era por supuesta incompetencia o por percibirse como agente dañino para sus hijos: "Estoy demasiado loco para cuidar de mi hijo", "He sufrido mucho que no quiero pasarle ese sufrimiento" (sic). Asimismo, quienes fungen en la función materna siempre buscan a alguien con quien sentirse acompañados (ya por un familiar, ya por el clínico) para que ese otro le dé sentido a los momentos de lejanía con su bebé. Pero sobre todo en la gran necesidad de hablar: "siento como si me faltara algo", "no es justo que no me dejen estar al lado de mi hijo", dice un padre al clínico. Otra figura es la de algunas madres que podían estar reunidas con sus bebés de manera casi ilimitada que, en cambio, no parecían requerir de ese tercero. 
Una aclaración terminológica es importante. En este trabajo la noción "padres" se ocupa para nombrar al padre y a la madre en conjunto; en cambio se usará el singular padre para referir a este personaje únicamente; función materna referirá al cuidador primario encargado de maternizar. Cuidador secundario, por su parte, es una referencia a quienes sólo se encargan de trámites legales, de la situación económica, de ser interlocutores con hospital para otras cosas de orden administrativo, que se encuentran ausentes y que no maternizan. Así, por norma, mínimo dos personas están presentes en relación con un hijo en el hospital. En el dispositivo, una manera de pensar la situación consistió en nombrar a los involucrados a partir de sus funciones. Señalar que se pensaron los lazos en lo singular según la relación de cada uno con su correlato, como lo señalan las flechas de la Figura 1.

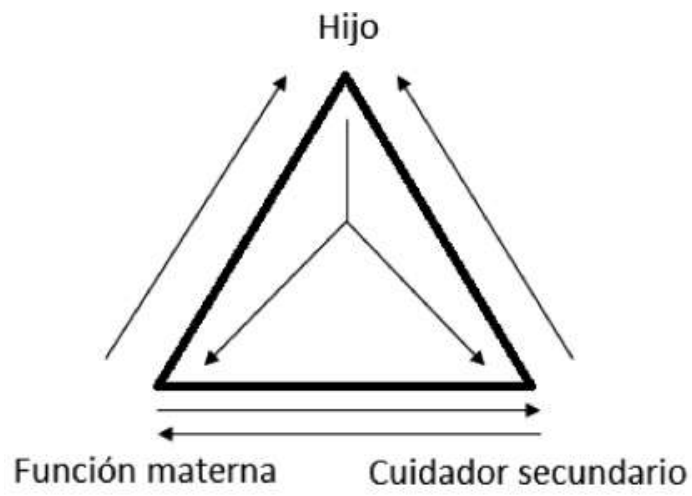

Figura 1. Trinomio.

En la figura se puede notar una línea que se desprende del hijo y se bifurca tanto hacia el cuidador secundario como hacia la función materna. Para el recién nacido es importante señalar que en él no se establece aún una distinción entre la función materna y el cuidador secundario, sino que devendrá, por una parte, a partir de las posibilidades de brindar placer mediante palabras y cuidados, y por otra parte en función del atemperamiento de las pulsiones que generan dolor en el recién nacido, como el hambre (Freud 2006/1895, p. 340). 
Hay que recalcar que siempre se notó una actitud distinta de parte de los neonatos para con quien hace la función materna y con su cuidador secundario. Así, se lograron observar cinco tipos de interacción con los cuidadores, padres o enfermeros:

1. Los bebés que interactuaban y jugaban con los cuidadores guardaban buen humor para con estos, se notaba una mejoría en sus niveles de oxigenación y un incremento de su ritmo cardiaco.

2. Los bebés que se relajaban, disminuían el ritmo cardiaco, manteniendo buenos niveles de oxigenación y acurrucándose al contacto con el otro.

3. Los bebés que rechazaban la interacción de una persona, movilizándose mucho, incrementaban el ritmo cardiaco, pero disminuían el nivel de oxigenación; en general se veían incómodos.

4. Los bebés que rechazaban de manera generalizada las interacciones con las personas, mantenían niveles muy bajos de oxigenación y un alto ritmo cardiaco.

5. Los bebés que se notaban desinteresados del mundo y ya no respondían a las interacciones, constantemente tenían un ritmo cardiaco y nivel de oxigenación bajos.

Develar estas relaciones entre los cuidadores y el neonato, no solo permitió trabajar con el dispositivo clínico conformado desde el trinomio, sino que resultó útil para mostrar lo fundamental del entendimiento del cuidado especial en el recién nacido: del relajamiento al juego y viceversa.

\section{El recién nacido}

Para que exista una relación tendrá que eludirse el autismo. En efecto, para Lacan el niño autista está también en el lenguaje. Estos niños se sienten perseguidos por la presencia del Otro, especialmente por la vía de la voz y la mirada produciendo su anulación, desviando su mirada y rechazando la intimación con ese Otro, como lo señala Lujan (2014): 
Parte de la prematuración del hombre al momento de nacer, y toma como sustrato la noción de un cuerpo fragmentado por la inmadurez misma y la falta de dominio motriz. Estos elementos le permiten ubicar que, en un primer tiempo, el cuerpo del niño no es vivido como uno. (p. 6).

De la misma manera, no puede generar aún la imagen de su cuerpo como unificado ni distinguir las cualidades de los Otros fuera de las cuestiones nutricias y de contacto afectivo. Estos pedazos de cuerpo deberán simbolizarse como falta. La estructura del deseo y del goce se orientarán en torno a estos objetos por la vía de la escritura de la falta y de la separación de un resto del cuerpo propio que se tornará ajeno (p. 6). La relación que se establece del lado de la función materna se comprende a partir del deseo de la madre, a saber, desde el lugar de erotización, ya que el nutricio es cubierto por las enfermeras.

\section{Las ubicaciones del deseo de hijo desde Lacan}

A propósito de la enseñanza de Lacan como ubicaciones del niño, Laurent (1986) propone tres respuestas posibles desde el eje del deseo. Por un lado, el niño como falo de la madre: esto es, se afinca la relación por la dependencia de amor, "por el deseo de su deseo en cuanto que la madre misma lo simboliza en el falo" (p. 46); es decir, la madre ama para ser amada por su hijo: "yo sé que mi hijo me ama y yo estoy aquí por él". Por otra parte, el niño como síntoma: el niño viene como respuesta a la cuestión que plantea este enfrentamiento con el Otro. Así se configura como síntoma del deseo de la madre en cuanto que está articulado con el Nombre del Padre, lo cual produce una significación al deseo de la madre. A lo que agrega Laurent:

el síntoma del niño representa la verdad del discurso de la madre, no lo es del discurso de la madre como tal, sino de la verdad de la estructura de la pareja. Por ejemplo, aquellas madres resignadas:

"Dios me ha enviado un hijo así como castigo". (p. 48).

Por último, el niño como objeto del fantasma de la madre: el niño se convierte en el objeto del fantasma de la madre, el mismo que el objeto $a$. (pp. 45-51), lo que puede ilustrarse con madres con amenazas de suicidio. 


\section{Las funciones parentales}

En cuanto a la función paterna -para la geografía que nos interesa mostrar, función de quien funge como cuidador secundario- explica Laurent (2018):

Lacan define el nuevo padre a través de un fetichismo particular (...) del objeto que una mujer produjo. El niño como objeto $a$ de la madre en tanto que objeto real. De este objeto $a$, el padre debe tomar un cuidado particular, como se dice, "paterno". Este cuidado deja este hombre -que se ocupa de los objetos a de una mujer-, lo deja en lugar de síntoma. (s.p).

En cuanto a la función materna, fue articulada en tanto nutricia, depositaria del deseo sobre su hijo. Del hijo se señala que, aunque vivo o muerto, siempre dota a los otros de un nuevo vínculo y una nueva decisión para con él, formulando así las preguntas en los padres: ¿lo tomaré o no? y ¿cómo lo tomaré?

Dichas funciones solo podían aparecer si se proponía un espacio libre de la medicalización de la vida, espacio de convivencia que de manera excepcional no tuviera que ver con la salud de los agentes, sino propiamente de relacionarse entre ellos, respetando lo más singular de cada uno, situación abismalmente distinta de la sucedida bajo el resguardo médico-enfermeril.

Rumbo al final del acompañamiento, cuando los agentes señalados habían logrado crear lazos amorosos psíquicos entre ellos, se volvió propio hablar de complejo familiar siguiendo la articulación de Aromí (2005):

Para el psicoanálisis la familia es un tejido que permite que se envuelva un enigma. Un tejido de relaciones, de afectos y desafectos, pero sobre todo un tejido de palabras y de silencios que hacen que ese enigma sea transmisible de una generación a otra [sic]. (s.p.).

Dicho enigma tejido formula familias, pero destejido es capaz separarlas o aislarlas e impedir que se unan. Situación bastante cotidiana en el escenario hospitalario. 


\section{El dispositivo clínico de acompañamiento al trinomio. La casuística}

Para abordar las formas de satisfacción pulsional y síntomas se eligió trabajar con los neonatos hospitalizados por problemáticas asociadas a su salud o con sus cuidadores o con las personas que se veían afectadas y no eran el cuidador primario.

La realización de intervenciones de mirada psicoanalítica con las figuras parentales se encaminó a sostener el deseo de cada uno. La clínica psicoanalítica, aplicada a la terapéutica con neonatos, contribuyó a construir un maternaje con bebés que se encuentren en situación de hospitalización, mediante el cual se logró dar sostén afectivo y corporal buscando contribuir a su constitución psíquica en condiciones adecuadas. El dispositivo se propuso disminuir la angustia mediante un manejo que promueva la seguridad y el descanso. Se conformó con el paciente ya identificado mediante una serie de entrevistas con los padres o con las figuras que se presentaran a las entrevistas semanales, siguiendo una orientación psicoanalítica, y se puso en marcha en cada caso de manera específica con la clínica psicoanalítica orientada a la terapéutica con el bebé. También buscando conocer los tiempos viables en función de no irrumpir los momentos que pase con la madre, para de esta manera no ser un elemento de obturación del vínculo que se establece. En función de esto se determinaron las actividades específicas y posibles de acuerdo a la situación particular del bebé. Por otra parte, se mantuvo junto a la incubadora un escucha flotante para tratar de traducir qué es lo que solicitaba el bebé. Finalmente, se dio la aplicación directa de la clínica psicoanalítica para la primera aproximación a los bebés que no han podido ser recibidos (o no del todo) por sus figuras maternantes y presenten una imposibilidad para el sostenimiento de la vida y/o problemáticas psicofísicas por la falta de la presencia de una figura maternante (Otro).

En suma, esta clínica busca el sostenimiento del bebé a partir de la promoción de la constitución psíquica que le permita inscribir en su cuerpo una realidad subjetiva, para representar sus dolencias por la situación de separación en la que se encuentra y lograr representar de manera imaginaria y simbólica lo que desde el real insis- 
te. Esto es alcanzado mediante dos formas, la primera es a partir de la presencia constante y erógena del oído y la vista que permitan dar a conocer la existencia de una realidad simbólica en la cual se puede inscribir para poder llegar a escuchar su voz. La segunda es tocar y nombrar el cuerpo del bebé para ayudar en la construcción de la representación psíquica del cuerpo. Por medio del cuerpo a cuerpo y la palabra del acompañante se hace sentir la presencia de Otro que lo llama y le habla para que se inscriba en él el deseo de vivir. Además, se le buscan explicar al bebé las situaciones posibles de angustia en las cuales puede estar presente de manera física y que por lo tanto no sabe controlar, para que pueda representarlas como algo ajeno a sí mismo. La unión de los elementos anteriores parece por sí misma suficiente para revertir algunas enfermedades psicofísicas presentes.

Los casos de un recién nacido expósito en falla y el de una madre con su hijo recién fallecido, muestran más ampliamente el dispositivo de acompañamiento y el trabajo al trinomio.

En la Unidad de Cuidados Intensivos Neonatales (UCIN) del Hospital Materno Infantil de la Ciudad de San Luis Potosí, lugar donde se realizó dicha investigación, se visita a un recién nacido de ocho días, que tres días antes había ingresado a ese servicio. La madre, procedente de un rancho a cuatro horas de la ciudad de San Luis Potosí, lo llevó "porque el niño no tenía buen color, se le notaba pálido"; fue así que le diagnosticaron una malformación, palabra que se convertirá en un significante importante. Su esófago no estaba conectado con su estómago, así que harían una operación para unirlos.

El clínico se presenta con la madre para darle a conocer algunas formas en las que puede relacionarse con su hijo ya en incubadora y conectado a varios aparatos. Así, le muestro algunas formas de comunicación humana con neonatos que se pueden llevar a cabo a pesar de la aparatología que limita la interacción, explicándole, además, la relevancia de su estadía con él para continuar con el proyecto de constitución psíquica de su hijo. Responde que se ausentará por motivos económicos y para continuar con su familia en su lugar de origen, le 
pido que me permita asistir a su hijo en su ausencia, lo que ella acepta. La madre se ausentaba y lo visitaba tres o cuatro horas a la semana, conforme lo autorizado médicamente, es decir, lo equivalente a dos o tres días en el hospital.

A este bebé, posterior a su operación, se le mantenía conectado a un respirador artificial, al igual que a otras máquinas destinadas a medir sus signos vitales y otras cosas. Se encontraba en una habitación aislada y pequeña -dos por dos metros- en la misma UCIN, donde lo único que se escuchaba era el ruido que estas máquinas emitían. Se esperaba que el aislamiento impidiera las infecciones en las heridas abiertas por la operación.

En rigor, este pequeño paciente se encontraba limitado y en alto riesgo de comprometer su estructuración psíquica de importancia vital en los recién nacidos, como ya lo demostró Spitz (1974) con los síntomas de hospitalismo y depresión anaclítica, quien encontró una involución de las funciones del desarrollo en niños a quienes únicamente se les atendía desde cuestiones asistenciales, enfermeriles y sin afectos.

Visitándolo de lunes a viernes y a la misma hora, lo llamaba por su nombre, le tendía un dedo para que pudiera aferrarse a él. Con ese dispositivo, luego de varios días de insistir, este pequeño sujeto de quien no se esperaba nada y que no miraba ni prestaba atención a las personas que entraban a su habitación, pasó a ser alguien impaciente e inquieto sobre todo por mi tardanza; se manifestaba con baja saturación de oxígeno en su sangre mediante el aparato que medía los signos vitales y después de un rato de estar con él se estabilizaba. Asimismo, los días que lo visitaba mantenía constantes dichos signos, cuando llegaba el fin de semana y lo dejaba de ver, lo volvía a encontrar y me percataba que los signos se habían modificado teniendo grandes varianzas. Lo anterior se pudo constatar con las hojas de registro que mantenían las enfermeras.

En cuanto a las operaciones, el primer intento de unión del esófago y el estómago falló. Perforaron un pulmón y así parte de la leche 
y saliva que corrían por su garganta iban a dar a su pulmón, lo cual complicó su salud y tuvieron que suspender la alimentación otra vez, esto lo mantenía irritable. Los médicos tuvieron que colocar un sello al pulmón por donde extraían los líquidos y aparecieron algunas infecciones que fueron tratadas con bastantes antibióticos.

Todos los dolores que ese bebé padecía intentaban ser subsumidos por medio de fármacos para el dolor, su eficacia era de treinta minutos y se administraban cada seis horas. Fue ahí donde mi presencia y los cuidados proporcionados le permitían descansar de sus dolores. Lo arrullaba de la manera que se me permitiera, a veces tocándolo con la voz, otras tocándolo con las manos.

Cada sesión entraba, miraba cómo se encontraba, parecía darme una serie de quejas, lloraba, me mostraba su rostro con dolor. Yo iniciaba a cantarle y acariciarlo hasta que durmiera, me quedaba ahí cantando y a la espera de que despertara, muchas veces no lo hacía y me retiraba. Era el canto el que de verdad paliaba el dolor de ese bebé, puesto que después de mi intervención podía dormir hasta dos o tres horas de corrido. Su humor mejoraba y se le notaba más al tanto del mundo, ya no tan abstraído en sí mismo, a su vez los signos vitales tomados por las enfermeras se mantenían constantemente altos.

La madre empezó a visitarlo con mayor constancia cuando le dijeron que ya habían retirado el sello -la válvula al pulmón ya descrita- y que la operación del esófago al estómago ya había sanado; así también cuando la malformación había sido revertida y su economía psíquica y monetaria ya no le era tan adversa, de lo último puesto que había conseguido que algunos familiares le aportaran cierta cantidad de dinero semanal mientras salía del hospital su hijo.

El segundo caso para ilustrar el acompañamiento al trinomio es de una madre con su hijo, recién fallecido, y que ya se ha citado previamente. Es aquella adolescente que no lograba expulsar de su vientre al hijo muerto, a pesar de la suministración masiva de medicamentos para ese fin. Se atiende a la paciente en el Área de Vigilan- 
cia Obstétrica del mismo hospital (AVO), sala que comparte con tres pacientes más y cuya única privacidad entre ellas es por medio de cortinas corredizas.

La joven proviene de una familia que padece diabetes y dice que sabía muy bien cómo tenía que descuidarse para contraerla, actos que llevaba deliberadamente a cabo desde su infancia: "mis padres nunca me trataron bien, nunca me sentí parte de esa familia donde no tenía un lugar", lo que hacía mantener desde su infancia pensamientos de suicidio. Recién se había mudado con su novio iniciando una vida de pareja. Sin embargo, durante su hospitalización se oponía a verlo, rechazándolo en los horarios de visita, pues sentía que le había fallado. Comenta que él la había tratado muy bien desde que salió huyendo de la casa de sus padres, cuestión que la llevó a "salir de esa depresión de años"; ese periodo coincide con el momento de su embarazo, se sentía libre y nunca se había cuidado en ningún sentido ni para evitar embarazarse ni para controlar la diabetes. El embarazo había sido bien recibido por su pareja, pero ahora que se había interrumpido y no sabía cómo darle la cara.

En consulta con un primer médico, dado que se sentía mal, le reveló su embarazo, pero por su condición diabética y sus altos niveles de glucosa en la sangre, se anunciaba de alto riesgo y con muy altas posibilidad de perderlo. Se le recomendó cuidarse y prepararse con antelación para su próximo embarazo, como una orden prescriptiva.

Hospitalizada en el Área de Vigilancia Obstétrica (AVO), observaba y maldecía en voz baja a las demás madres que se encontraban en la habitación, las cuales aún tenían a sus hijos vivos, diciendo tenerles una gran envidia. Con el hijo muerto dentro de su vientre, dice, "perdí todo lo que me daba una esperanza en la vida", que era el hecho de ser madre de alguien a quien podría cuidar, por quien valdría la pena vivir y a su vez significaba darle ese hijo al hombre que amaba y que la trataba tan bien.

Hablar que ahí había una madre que necesitaba ser nombrada como tal, que había una mujer que amaba, que no podía dar la cara 
por sentir que había fallado (aunque su pareja no la culpabilizara), madre, en fin, que cargaría junto a ese padre a un hijo, fenecido y ya nombrado, fue sustancial, más allá de hablar de un hijo como producto o como un embarazo de riesgo que se había interrumpido. Así, señalando la existencia de lazos amorosos que, ahora tejidos, envolvían el enigma de la vida y de la muerte, se permitía la aparición de una familia. Es el deseo imaginado de su hijo tan amado y ahora fallecido lo que los une. Al finalizar la sesión se pudo notar en ella una sonrisa, la cual, al momento de percatarse, borró de manera inmediata, pero su rostro no pudo ser el mismo otra vez, los rastros de esa sonrisa impedían la cara de enojo que pretendía poner. Es allí que la reintroducción del deseo de un hijo, quien, en palabras de la paciente, "también hubiera deseado lo mejor para ella", junto con el deseo de su pareja, fueron los elementos con los que se terminó la sesión. Esa tarde pudo expulsar al hijo muerto. Ahora lo llevaba simbólicamente, y ya no era necesario guarecerlo. Al siguiente día la paciente ya no se encontraba en el Área de Vigilancia Obstétrica (AVO).

Lo sucedido pudo ser comprendido desde el movimiento de su hijo como objeto, transfiriéndolo del plano imaginario al plano simbólico. A propósito del objeto, Brousse (2010) plantea que: “(...) En psicoanálisis [de orientación lacaniana], cuando se habla de objeto, se habla de un objeto perdido (...)" (s.p.), y sostiene lo siguiente:

Lacan (...) opone objetividad con otro término que inventa: "objetalidad". Ubica la objetividad del lado de los objetos exteriores, de los objetos que están en el espacio, y la objetalidad para definir estos raros objetos que están perdidos desde el comienzo. Por lo tanto, solo tenemos acceso a ellos a través de representaciones o huellas. (s.p.).

La reintegración de la existencia de ese hijo, el juicio de existencia que la volvió madre, la creación de un objeto interior que ya no existía como debería en el exterior permitió establecer un lazo desde otra posición. El perder a su hijo, ahora sin vida dentro de su vientre, y llevarlo al plano de lo simbólico, fue lo que permitió recuperarlo, así como salir del atolladero en el que se encontraba. 


\section{Conclusiones}

La infancia se encuentra en una constante transformación, generando cultura, perspectivas, formas de ver, observar e intervenir. Etimológicamente la palabra infancia viene del latín y significa "el que no habla", pero, ¿no habla o no se le escucha? La infancia consiste en ser considerado "sin voz", lo que parece no validar lo que de ahí proviene y no hacer un intento por adecuar la escucha. De ahí que en lugares como este vendrán varios términos inventados de Lacan, el pasaje del paciente, aquel que espera, aquel que padece, al de analizante, personaje activo dentro una situación analítica, aquel que asocia libremente, que busca hacer cambios, modificar su situación. He aquí una primera distinción, entre estar junto a aquel "sin voz" y aquel "pequeño analizante", lo que se modifica es la posición del analista frente a él. En la primera posición se da por sentado que no hay nada que escuchar, en la segunda se busca crear dispositivos o disposiciones que permitan entender ese decir.

Sostenido en lo anterior, se observó en el hospital materno infantil donde se llevó a cabo la intervención, que la relación médica dirigida al paciente, dispuesta para atender los signos de enfermedad o mal funcionamiento orgánico, de manera amable da por oídos (pero no escuchados) los síntomas de los pacientes, sin poder hacer algo con eso, ya que la disciplina médica como tal no lo contempla.

Los tratamientos consisten en dar a conocer a los pacientes su situación, dando por sentado que ellos la entienden, responsabilizándolos y solicitando de ellos el autocuidado. Pero las unidades de cuidado intensivo (UCI) son la excepción, puesto que se estima que los pacientes no pueden cuidar de sí mismos. En ese escenario, es asî que se involucra un amplio número de enfermeros dispuestos a generar los cuidados orgánicos requeridos, aunado a una observación constante de un médico responsable de la unidad. Observan y consideran al paciente como un individuo al que se le busca mejorar su salud; gran parte de su desconcierto viene de los momentos en que se vuelve evidente lo contrario. La puesta en marcha de un psicoanálisis aplicado a la terapéutica sobre el trinomio en un escenario hospita- 
lario presenta grandes dificultades. Se trata de variadas vicisitudes cotidianas en los hospitales que demandan ser atendidas. El trabajo ha mostrado la experiencia psicoanalítica analizando y desplegando dicha intervención, al mismo tiempo da cuenta de las demandas en el hospital, acentuando el acto de tratamiento y las reflexiones ocasionadas y, particularmente, considerando el tratamiento de lo real y el goce implicado en el síntoma. Se ha intentado ser el escenario del paciente que, al entrar a la dinámica del hospital, sufriente por los avatares de la cotidianidad, se inserta en una experiencia silenciada y obturada, para permitir, más bien, que en dicho escenario se desplieguen todas las formas posibles de expresión de aquello que pugne por encontrar medio de lenguaje. Con esto, se apunta a que las intervenciones desde el analista permitan que dicha parte reprimida pueda encontrar nuevas formar de transitar, haciendo registro en el cuerpo de un sujeto.

Las huellas subjetivas en esas situaciones expuestas a la medicalización siempre estarán en el psiquismo, lugar donde no existen las borraduras sino únicamente las reediciones de esos mismos capítulos de la vida. El neonato en la UCIN requiere no únicamente de los cuidados médicos, sino de una parentalidad y de sus funciones para mantenerse en la vida. La función del psicoanalista consiste en colocarse como cuidador primario, conservando funciones maternizantes. En cuanto a la parentalidad, se puede leer desde las marcas psíquicas que se generan desde lo real, como se planteó en el caso de la paciente que sintió a su hijo, esto lo gestó psíquicamente y ella le dio acogida promoviendo estos nuevos lugares, padre-madre-hijo.

El enfrentamiento con eso innombrable (Real) produce consecuencias clínicas e institucionales de las que aquí se propuso dar cuenta. Los mecanismos disciplinares militarizados en el hospital, por señalar a la manera de Foucault, las acciones medicalizantes como ideología de cura, tienden a considerar a la persona como un sujeto orgánico únicamente, sujeto de políticas públicas donde lo demás no hace sentido. La investigación encontró argumentos que refutan el hecho de mantener lejanos a los padres de su hijo en la UCIN, ya que el argumento de "evitar infecciones", además de ser discutible, dificulta de manera importante el desarrollo de la constitución psíquica del bebé. 
Es la inclusión del psicoanalista lo que puede introducir la relación epistemosomática y una nueva forma de concebir al hombre. De allí que sea de gran importancia iniciar discursos multidisciplinarios o transdisciplinarios para formular una atención distinta a los pacientes. Lo anterior provoca una premisa fundamental del psicoanálisis aplicado: continuar con la reflexión y la problematización tanto de la clínica como de la teoría psicoanalítica.

\section{Referencias}

Anders, V. et al. (s.f.). Etimología de televisión. Recuperado de: http://etimologias.dechile.net/?auscultar

Aromí, A. (2005). ¿Quées una familia? Recuperado de: http:/ / www.nel-mexico.org/index.php?sec=Actividades-internacionales\&file=Actividadesinternacionales/Textos-Conferencias/14-09-05_Anna-Armoni.html

Brousse, M-H. (2010). Objetos extranjeros, objetos inmateriales. Revista La lúnula del CIEC. Recuperado de: http://www.cieccordoba.com.ar/lunula/leermas17.html.

Freud, S. (2006/1895). Proyecto de psicología. En J. Strachey (Ed.) y J.L. Etcheverry y L. Wolfson (Trads,). Obras Completas (Vol. I, p. 340). Buenos Aires, Argentina: Amorrortu.

Lacan, J. (1966). Psicoanálisis y medicina. El lugar del psicoanálisis en la medicina

Recuperado de: https://www.lacanterafreudiana.com.ar/2.5.1.9\%20\%20 \%20PSICOANALISIS\%20Y\%20MEDICINA,\%201966.pdf

Laurent, É. (1986). El niño y su madre. En El Analiticón (Vol. 1, 45-52). Barcelona, España: Ediciones Paradiso, Correo del Campo Freudiano en España.

Laurent, É. (18 de mayo de 2018). Los niños de hoy y la parentalidad contemporánea. Conferencia en la Facultad de Psicología UBA, Buenos Aires, Argentina. Recuperado de: https://www.youtube.com/ watch? $v=j-Y 89$ V6ofHo.

Lujan, I. (2014). Curso Clínica con niños. Unidad 2: Hacerce de un cuerpo. Dictado en UCES: Universidad de Ciencias Empresariales y Sociales a través de UCES virtual.

Miller, J. (2012). Embrollos del cuerpo. Buenos Aires, Argentina: Paidós

Orueta Sánchez, Ramón, Santos Rodríguez, Coral, González Hidalgo, Enrique, Fagundo Becerra, Eva Mª , Alejandre Lázaro, Gemma, Carmona de 
la Morena, Javier, Rodríguez Alcalá, Javier, Campo del Campo, José $\mathrm{M}^{\mathrm{a}}$ del, Díez Andrés, María Luisa, Vallés Fernández, Natalia, \& Butrón Gómez, Teresa. (2011). Medicalización de la vida (I). Revista Clínica de Medicina de Familia, 4(2), 150-161. Recuperado en 24 de febrero de 2020, de http:/ / scielo.isciii.es/scielo.php?script=sci_arttext\&pid=S1699-695X20 $11000200011 \& \operatorname{lng}=\mathrm{es} \& \operatorname{lng}=\mathrm{es}$.

Spitz, R. (1974). El primer año de vida del niño. México: Fondo de Cultura Económica.

Giangaspro, E. (2016). Algunos apuntes sobre la medicalización de la existencia. En B. Udenio (Ed.) iA la salud de todos! Niños saturados Revista El Niño, 14, pp. 21-27 Olivos, Argentina: Grama Ediciones. 